\title{
Realizing the Visualization of Plane Wave in Conductive Medium by MATLAB Graphics Technology
}

\author{
Lifen Li \\ Yanching Institute of technology, Sanhe, Heibei, China 065201 \\ lilifen66@aliyun.com
}

\begin{abstract}
Keywords:Matlab, Simulation, Uniform Plane Wave
Abstract:The matlab programis usuarallyused to make simulation of electromagnetic distribution and the transmission of waves, which dynamiclyshows the propagation of waves. This article focuses on simulating the dynamic process of the propagation, reflection andrefraction of uniform plane wave in a different medium.
\end{abstract}

\section{Introduction}

The electromagnetic fields and waves is a kind of compulsory basic course of undergraduates withelectronic communication major, which mainly researches the basic properties and rules ofelectromagnetic fields and waves, in order to solve some practical engineering problems, so thatthey can be closely co-ordinated with microwave technology and antenna technology courses. Inthe teaching process, students generally reflect that the course is theoretical, and the concepts areabstract, and it contains a large number of mathematical formulas, leading to a largeamount of computation. Whether electromagnetic fields or waves, they are both invisible and cannot be touched. The fact is that teachers feel hard to teach while students feel hard to understand iscurrently a common phenomenon.Because of matlab's powerful features and visual simulationenvironment, it provides simulation conditions for teaching electromagnetic fields and waves.Matlab has a large number of two-dimensional and three-dimensional graphics functions, whichhelps students understand and master the law of electromagnetic fields and waves propagation. Inthis paper, the use of matlab for the simulation of propagation, reflection and refraction of uniformplane wave in a different medium has achieved good teaching results.

\section{The propagation of uniform plane wave in a conductive medium}

Let's the propagation of electromagnetic waves along the $z$-axis direction in the planeperpendicularto the z-axis, the points have the same intensity of electromagnetic field amplitude and direction ofvibration, i.e., only the size of the electric and magnetic fields related to $\mathrm{z}$, and it independent of $\mathrm{x}$ andy, so the electromagnetic wave is uniform plane wave.

\subsection{The simulation processes of electromagnetic conductive medium}

Uniform plane wave in the sea simulation's process is as follows,accordingto the known parameters, calculate the number of $\sigma / \omega \varepsilon$ to judge themediumis good or bad conductor. And then calculate the number of $\boldsymbol{\alpha}, \boldsymbol{\beta}, \boldsymbol{\eta}, \boldsymbol{\varphi}$. Settingthe forloop takes $\mathrm{T}$ as the independent variable. The instantaneous value of the electric fields and magnetic fields are written in the loop.Calling the matlabmeshgrid, plot3, pause and other functions, to simulate thegraphics of electric and magneticfields.

\subsection{The simulation of waves of different frequencies in the same medium}

Set a uniform plane with a frequency of $\mu=\mu_{0}, \boldsymbol{\varepsilon}=81 \varepsilon_{0}, \sigma=4 \boldsymbol{S} / \boldsymbol{m}$ as an example, let'sassume $\overrightarrow{\boldsymbol{E}}=\overrightarrow{\boldsymbol{e}}_{\boldsymbol{x}} \boldsymbol{E}{ }_{\boldsymbol{x}}$ and the amplitude is $1 \boldsymbol{V} / \boldsymbol{m}$ 。

\subsubsection{Transmission of $f=1 \mathrm{kHz}$ in conductive medium}

When $\boldsymbol{f}=1 \mathrm{kHz}, \frac{\sigma}{\omega \varepsilon}=\frac{\sigma}{2 \pi f \varepsilon_{0} \varepsilon_{r}}=8.9 \times 10^{5}>>1$, sothe sea water is a good conductor.then $\alpha=\sqrt{\frac{\omega \mu \sigma}{2}}=0.04 \pi \mathrm{Np} / \mathbf{m}, \boldsymbol{\beta}=\sqrt{\frac{\omega \mu \sigma}{2}}=0.04 \pi \mathbf{r a d} / \mathbf{m}, \quad \delta=7.9577 \boldsymbol{m}$, Therefore, the transient expression of 
electric fieldis $\overrightarrow{\boldsymbol{E}}(\mathbf{z}, \boldsymbol{t})=\overrightarrow{\boldsymbol{e}}_{\boldsymbol{x}} \times \boldsymbol{e}^{-0.04 \pi \boldsymbol{z}} \cos \left(2 \pi \times 10^{6} \boldsymbol{t}-0.04 \pi \boldsymbol{z}\right)$, while the transient expression of magnetic field is $\overrightarrow{\boldsymbol{H}}(\mathbf{z}, \boldsymbol{t})=\overrightarrow{\boldsymbol{e}}_{\boldsymbol{y}} 0.71 \boldsymbol{e}^{-0.04 \pi} \cos \left(2 \pi \times 10^{6} \boldsymbol{t}-0.04 \pi \boldsymbol{z}-45^{\circ}\right)$, a time waveform of theectromagnetic wave shown in Fig1,theelectric fields strength is the vertical and the magnetic is horizontal. By graphics can visually see the electric and magnetic fields amplitude is attenuated.
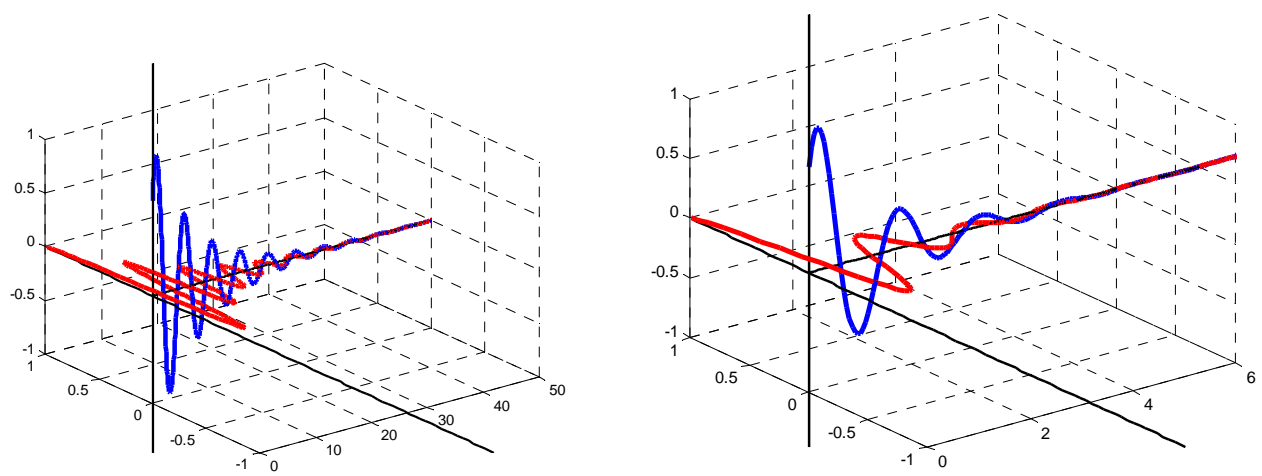

Fig 1 the transmission of $\boldsymbol{f}=1 \mathbf{k H z}$ Fig 2 the transmission $\boldsymbol{f}=1 \mathbf{M H z}$

\subsubsection{Transmission of $f=1 \mathrm{MHz}$ in conductive medium}

When $f=1 \mathrm{MHz}, \frac{\sigma}{\omega \varepsilon}=\frac{\sigma}{2 \pi f \varepsilon_{0} \varepsilon_{r}}=890>>1$, sothe sea water is a good conductor. Sothe situation is $\alpha=\sqrt{\frac{\omega \mu \sigma}{2}}=1.26 \pi \mathrm{Np} / \mathbf{m}, \quad \beta=\sqrt{\frac{\omega \mu \sigma}{2}}=1.26 \pi \mathbf{r a d} / \mathbf{m}$, Therefore, the transient expression of electric fieldis $\overrightarrow{\boldsymbol{E}}(\mathbf{z}, \boldsymbol{t})=\overrightarrow{\boldsymbol{e}}_{\boldsymbol{x}} \times \boldsymbol{e}^{-1.26 \pi} \cos \left(2 \pi \times 10^{6} \boldsymbol{t}-1.26 \pi \boldsymbol{z}\right)$, while the transient expression of magnetic field is $\vec{H}(z, t)=\overrightarrow{\boldsymbol{e}}_{\boldsymbol{y}} 0.71 \boldsymbol{e}^{-1.26 \pi z} \cos \left(2 \pi \times 10^{6} \boldsymbol{t}-1.26 \pi \boldsymbol{z}-45^{\circ}\right)$. At a certain time, the electromagnetic wave is shown in Fig2, By graphics can visually see the electric and magnetic fields amplitude is attenuated and the electromagnetic wave is still TEM wave, electric field and magnetic field is in the phase difference, and the amplitude of the electric field and the magnetic field is attenuated by the transmission distance, and the propagation distance in the sea is shorter.

\subsubsection{Transmission of $f=100 \mathrm{MHz}$ in conductive medium}

When $f=100 \mathrm{MHz}, \frac{\sigma}{\omega \varepsilon}=\frac{\sigma}{2 \pi f \varepsilon_{0} \varepsilon_{r}}=8.90$, sothe sea water is normal conductor. Then the situation is $\boldsymbol{\alpha}=\boldsymbol{\omega} \sqrt{\varepsilon \boldsymbol{\mu}}\left\{\frac{1}{2}\left[\sqrt{1+\left(\frac{\sigma}{\omega \varepsilon}\right)^{2}}-1\right]\right\}^{\frac{1}{2}}=11.97 \pi \mathrm{Np} / \boldsymbol{m}, \boldsymbol{\beta}=\boldsymbol{\omega} \sqrt{\varepsilon \boldsymbol{\mu}}\left\{\frac{1}{2}\left[\sqrt{1+\left(\frac{\sigma}{\omega \varepsilon}\right)^{2}}+1\right]\right\}^{\frac{1}{2}}=42.1 \mathrm{rad} / \boldsymbol{m}$. The transmission of wave is shown in Fig3, compared with Fig1 and Fig2, the electric field and the magnetic field amplitude decay faster and faster.
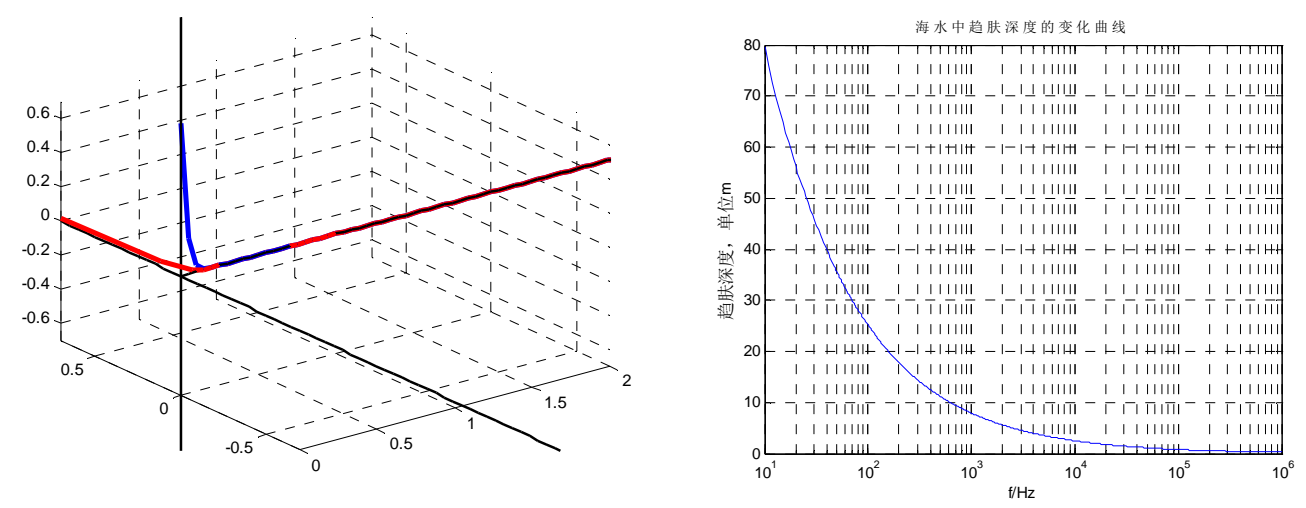

Fig 3 the transmission of $f=100 \mathrm{MHz}$ Fig 4 the curve of the skin depth in the sea water 


\subsubsection{Transmission of $f=10 \mathrm{GHz}$ in conductive medium}

$$
\text { When } f=10 G H z, \frac{\sigma}{\omega \varepsilon}=\frac{\sigma}{2 \pi f \varepsilon_{0} \varepsilon_{r}}=0.089<<1, \quad \alpha=\frac{\sigma}{2} \sqrt{\frac{\mu}{\varepsilon}}=26.7 \pi, \quad \beta=\omega \sqrt{\varepsilon \mu}=600 \pi \text {, }
$$

The phase shift constant is the same as the ideal medium.the attenuation constant is independent offrequency, with the value increasing, the attenuation of electromagnetic wave is more rapidly than the situlation of $f=100 \mathrm{MHz}$. The same kind of a medium is dielectrics or conductors, is not only related with the medium parameters, but also related with the frequency, the higher value of the frequency, the quicker decreasing of the electromagnetic wave in the medium. From the above analysis, the submarine should choose low frequencyelectromagneticwave communication under the water.

\subsubsection{The simulation of waves with different frequencies in the same medium}

The calculation method of the skin depth is $\delta=1 / \sqrt{\pi f \mu \sigma}=1 / \sqrt{\pi \times 4 \pi \times 10^{-7} \times 4 \times f}=251.6461 / \sqrt{f}$,

with the frequency range from $10 \mathrm{~Hz}$ to $1 \mathrm{MHz}$, the curve of the skin depth shown in Fig 4 . What can be seen from the figure is that with the increasing of the frequency, the decreasing of electromagnetic wave is faster in seawater, Therefore, inorderto maintain lower attenuation, the frequency of electromagnetic wave must be lower.

\subsection{The transmission of the same frequency in different medium}

When a uniform plane wave transmissing in the copper,which electrical parameters is $\boldsymbol{\sigma}=5.7 \times 10^{7} \mathbf{S} / \mathbf{m}$ and it is known to the wave propagation along the z-axis positive direction,letsthe formula is $\overrightarrow{\boldsymbol{E}}=\overrightarrow{\boldsymbol{e}}_{x} \boldsymbol{E}_{x}$ and the amplitude is $1 \mathrm{~V} / \mathrm{m}$, sothe value of $\frac{\sigma}{\omega \varepsilon}, \boldsymbol{\alpha}$ and $\beta$ is as follows, $\frac{\sigma}{\omega \varepsilon}=\frac{\sigma}{2 \pi f \varepsilon_{0}}=1.04 \times 10^{15}>>1{ }^{\alpha}=\sqrt{\frac{\omega \mu \sigma}{2}}=151 \pi N \boldsymbol{p} / \mathbf{m}, \boldsymbol{\beta}=\sqrt{\frac{\omega \mu \sigma}{2}}=151 \pi \mathbf{r a d} / \mathbf{m}$ and the skin depth is $\delta=0.0021 \boldsymbol{m}$.The transmission distance of electromagnetic waves in copper, is only 1/1000 of which in the sea.so the attenuation of electromagnetic wave decreases faster in a good conductor.the greater of the conductivity, the shorter of the transmission distance.Therefore, the transmissionof the electromagnetic waves is confined with the area near the surface of the conductor. When the media is the ideal conductor, the electromagnetic waves cannot enter inside of it.

\section{Vertical incident electromagnetic wave in a conductive medium}

When a uniform plane electromagnetic wave from a conductive medium incident to another in aconducting medium, the phase constant $\mathrm{k}$ is complex, therefore, the electromagneticwave is decreasing not only in the medium 1 but also in the medium 2, and the speed of attenuation is different, and the phase constants are different,too.Fig5 defines the simulation of vertical incidence electromagnetic wave in aconductive medium.

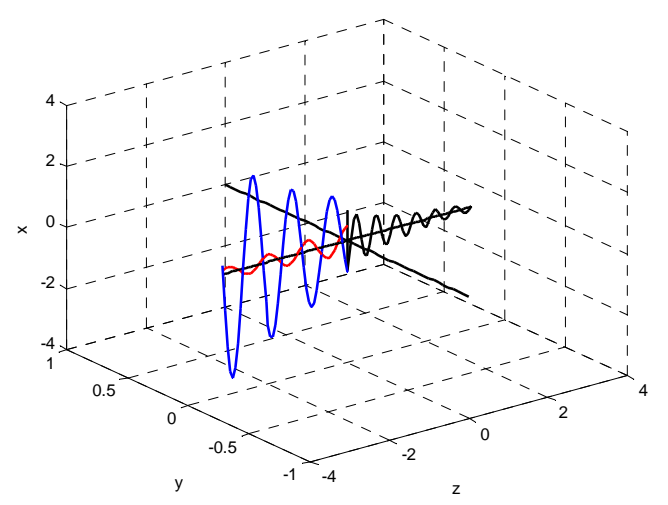

Fig5Vertical incident electromagnetic wave in a conductive medium

\section{Conclusion}

Using the drawing function of matlabrealizing the simulation of the abstract and invisible content in 
electromagnetic fields and waves,which can help students understanding the curriculum and the teaching content.Meanwhile it can trainthe ability of the students in designing, writing, and debuging the program. In this paper,

throughmatlab software to simulate the dynamic process of the propagation, reflectionand refraction of uniform plane wave in a different medium, which has enriched the teachingcontent, and has stimulated students' interest in learning.We have achieved good teaching results.

\section{Acknowledgement}

This article was financially supported by the project of Hebei Institute of Humanities and Social

Sciences Education Planning Project (Grant NO. GH152022).

\section{References}

[1] Mingjun Wang, Yingle Li, Jing Tang. The Application of MATLAB in the ElectromagneticFields and waves Teaching Curriculum [J]. Journal of Xianyang Normal University, 2009, 24(2): 89-91

[2] WeihuaZong, XiaoyunQu, Ying Wang, etc. The Application of MATLAB in the

Electromagnetic Fields and Waves Experimental Teaching[J]. Laboratory research andexploration, 2010, 29 (1): 53-56

[3] XiuliLv, HaiweiMou, Xianli Li, Research and Exploration of the Application of theExperimental Teaching of Electromagnetic Fields and Waves[J]. 2010, 29 (1): 110-112

[4] PengZou, Xiaoping Zhou. Electromagnetic fields and waves[M]. Third edition.Beijing:Higher Education Press, 2008

[5] Xiangyu Cao, Jun Gao, YueshengZeng, etc. Electromagnetic fields and waves [M], Xi'an:Xi'an Electronic and Science University press, 2007 